\title{
Solid State Replacement of Rotating Mirror Cameras
}

A. M. Frank, J. M. Bartolick

August 30, 2006

27th International Congress on High Speed Photography \& Photonics

Xi'An, China

September 17, 2006 through September 22, 2006 
This document was prepared as an account of work sponsored by an agency of the United States Government. Neither the United States Government nor the University of California nor any of their employees, makes any warranty, express or implied, or assumes any legal liability or responsibility for the accuracy, completeness, or usefulness of any information, apparatus, product, or process disclosed, or represents that its use would not infringe privately owned rights. Reference herein to any specific commercial product, process, or service by trade name, trademark, manufacturer, or otherwise, does not necessarily constitute or imply its endorsement, recommendation, or favoring by the United States Government or the University of California. The views and opinions of authors expressed herein do not necessarily state or reflect those of the United States Government or the University of California, and shall not be used for advertising or product endorsement purposes. 


\title{
061-A.M.Frank-Solid State Replacement of Rotating Mirror Cameras
}

\section{Solid State Replacement of Rotating Mirror Cameras}

\author{
Alan M. Frank ${ }^{1} \&$ Joseph M. Bartolick \\ Lawrence Livermore National Laboratory ${ }^{\mathrm{i}}$ \\ POB 808, Livermore CA, 94551-0808
}

\begin{abstract}
Rotating mirror cameras have been the mainstay of mega-frame per second imaging for decades. There is still no electronic camera that can match a film based rotary mirror camera for the combination of frame count, speed, resolution and dynamic range. The rotary mirror cameras are predominantly used in the range of 0.1 to 100 micro-seconds per frame, for 25 to more than a hundred frames. Electron tube gated cameras dominate the sub microsecond regime but are frame count limited. Video cameras are pushing into the microsecond regime but are resolution limited by the high data rates. An all solid state architecture, dubbed 'In-situ Storage Image Sensor' or 'ISIS', by Prof. Goji Etoh ${ }^{\text {ii }}$, has made its first appearance into the market ${ }^{\text {iii }}$ and its evaluation is discussed.

Recent work at Lawrence Livermore National Laboratory has concentrated both on evaluation of the presently available technologies and exploring the capabilities of the ISIS architecture. It is clear though there is presently no single chip camera that can simultaneously match the rotary mirror cameras, the ISIS architecture has the potential to approach their performance.
\end{abstract}

\section{Background}

It is useful to compare camera architectures by examining the limiting functions unique to high-speed imaging. These are, detection, shuttering and data transport. Rotating mirror cameras, with film as the detector, use the mirror both as the shutter and to move the image from one frame to the next. Framing rate is limited by the explosion speed of the mirror. Video cameras use the raster scan both, to limit the collection time and to transport the data, thus are limited by electronic bandwidth. Tube gated cameras are either single frame cameras ganged together through beam splitters or electronically deflected to different regions of a common detector. Tube cameras are thus frame count limited by dividing either the incoming light or the detector area respectively.

Edweard Muybridge, the grandfather of high speed imaging, used an array of sequentially triggered single frame cameras, to record the motions of a horse, in the 1870's. In the 1880's, J.E.Marey ${ }^{\text {iv }}$ introduced the concept of moving film cameras. Rotating mirror cameras were first invented in 1893, by C.V. Boys $v$. They were used initially only for streak cameras. Rotating mirror framing cameras did not emerge until 1939, when Charles Miller ${ }^{\mathrm{vi}}$ suggested focusing an image on the surface of the rotating mirror, then refocusing the image onto the film through an arc of multiple relay lenses (Fig. 1). The concept was put into practice by Berlin Brixner, ${ }^{\text {vii }}$ during the Manhattan Project. Two of his designs, the Models 6 and 189 cameras are still in use today. Many other high speed designs have been built but the asynchronous Mod 6 and the synchronous Mod 189 remain the basic models of the cameras used today.

1 frank2@llnl.gov; phone 1925422 7271, fax 19254222382 


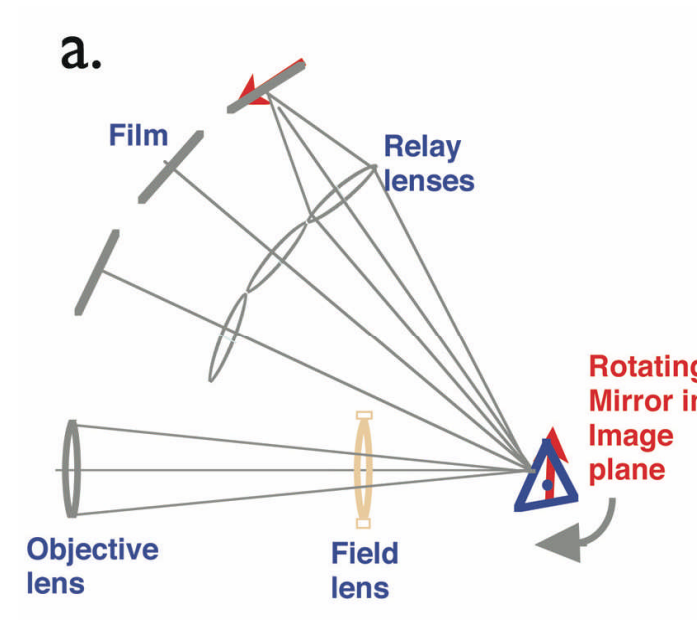

Fig 1.a. Schematic of a synchronous rotating mirror framing camera. b. Photo of the interior of a Cordin 121.

The Model 6 was manufactured at both Los Alamos \& Livermore Labs and perhaps by others, but never commercially. It is a 170 frame pseudo-continuous access camera with a frame size of $10 \mathrm{~mm}$ by $12.5 \mathrm{~mm}$, on $35 \mathrm{~mm}$ film. There is a gap of 8 frames due to internal shadowing. A synchronization system was added later to permit the placement of this gap in time. The maximum speed is $3.3 \times 10^{6}$ FPS with a limiting aperture of $\mathrm{f} / 32$.

The Model 189 was licensed to Beckman and Whitley and produced commercially by them from the late 1950 's until Cordin absorbed them in the early 1970 's $\mathrm{siii}$. It was a 25 frame synchronous camera capable of up to $4.0 \times 10^{6} \mathrm{FPS}$, although the frame size was only $8.5 \mathrm{~mm}$ by $19 \mathrm{~mm}$ at that speed. At $1.0 \mathrm{X} 10^{6} \mathrm{FPS}$ the frame size was $19 \mathrm{~mm}$ by $25 \mathrm{~mm}$. The limiting aperture was either $\mathrm{f} / 17$ or $\mathrm{f} / 21$, depending on the shutter stops used. The camera used $35 \mathrm{~mm}$ film. There was also a "B" model, a 120 frame version that became Cordin's Model 119. The Cordin 119 can record up to $25 \times 10^{6}$ FPS although the frame size is only $3.8 \mathrm{~mm}$ by $11 \mathrm{~mm}$.

The most recent upgrade, to the synchronous cameras, were designed in the 1970's, by Larry Shaw, at Lawrence Livermore National Laboratory. This camera was licensed to Cordin and became the Model 121. The camera used $70 \mathrm{~mm}$ film to increase the image size to $33 \mathrm{~mm}$ by $60 \mathrm{~mm}$ giving significantly improved image detail, with a maximum of $2.5 \times 10^{6}$ FPS for 25 frames. The Cordin 121 remains the mainstay of highspeed frame imaging at Livermore.

The speed of rotating mirrors is limited by both the strength of the mirror material and by the speed of sound. If either of these are exceeded the mirror will come apart, destroying the camera. The fastest cameras use helium turbines the drive beryllium mirrors to 10,000 revolutions per second. Synchronization is achieved with an optical detector sensing the phase of the mirror rotation. The detector signal is then used to trigger the event. Synchronization of multiple cameras is difficult, requiring automated gas controls for the turbines. As the mirror continues to rotate an external shutter is required to prevent over writing images.

Resolution of the cameras is limited by the dynamic distortion of the mirror. It is typically 10-15 line pairs per millimeter. The dynamic range is dependant on the film used. It is about 100 for fast color films and can be greater than $10^{4}$ with black \& white films. Sensitivity is generally limited by high aperture ratios of the optics, $\mathrm{f} / 17$ at best and often $\mathrm{f} / 32$. Also optical transmission through the optics is rarely better than $50 \%$. Even with the most sensitive films, powerful illumination is required.

A shortcoming of the rotary mirror framing cameras is the fact that each frame passes through a different relay lens. It is difficult to manufacture lenses with precisely the same focal length or to mount them with 
the same orientation. Thus the images tend to dance around when viewed as a movie. This also requires each frame to be individually calibrated for accurate photogrammetry.

Practical considerations for the use of rotary cameras are significant. Most important is the rapidly declining availability of suitable films and the need for an in house processing facility. The cameras are large and heavy requiring special mountings. They must be operated remotely in a beryllium proof, armored enclosure to protect against mirror mechanical failures. They also require large amounts of helium with sophisticated gas control systems. They are time consuming in loading and unloading film as well as film development.

The Cordin Company has developed a series of rotary mirror CCD cameras. Currently the Cordin $550^{\mathrm{ix}}$ is a new design rotating mirror camera with a mega-pixel CCD camera chip at each frame location in the film plane. It is capable of $4.4 \times 10^{6}$ FPS with up to sixty-two frames. The Cordin 510 derived from the Mod 189 $\&$ Cordin 119, has 128, frames of 300 kilo-pixels each with a maximum frame rate of $25 . \times 10^{6}$ FPS. The incorporation of solid state detectors into the rotary mirror architecture solves several former limitations. Electronic blanking eliminates the need for an external shutter to prevent overwriting and software can be used to correct for inter-frame misalignment.

\section{Electronic Frame Cameras}

To date no completely electronic camera offers the combination of speed, resolution, dynamic range and frame count offered by the rotary mirror cameras although several approaches have been closing in on the problem.

Traditionally camera development evolved independently for both the gating $\&$ image recording functions. Image intensifier tubes have been used with film for fast single frame recording for the last half century. The intensifier tubes have evolved through several generations. More recently, film has been replaced by solid state chips. For multiple frames, the Muybridge approach of separately triggered cameras is commonly used. The cameras may share a common line of sight through beam splitters, in which case the available light is divided between the cameras. Electron tubes with deflection plates can rapidly move an image to different locations on the output phosphor, creating a multi-frame camera. In this case, the resolution of the phosphor, film or chip, is divided by the number of frames recorded.

More recently solid state chip development has merged the gating \& recording functions. The light collection time of a CCD is approximately the time between successive readout functions. Thus if the chip can be read out very quickly it can become a high speed camera. The problem is that to approach megaframe readout the data transfer rates become prohibitive. Distributed parallel readout helps but currently, in a microsecond, only very few pixels can be read out drastically limiting resolution.

ISIS is a massively interlaced CCD architecture, which integrates the detection, shuttering and local data storage functions into a single chip. The basic concept of the architecture was described in a patent by Kosnocky \& Lowrance in $1994^{\mathrm{x}}$. Each photo site in the array is followed by a string of storage sites. On each clock cycle electrons are stepped from the photo site into and down the storage chain (Figure 2). Thus, a full frame is collected with each clock cycle. The inter-frame time is limited by thermal constraints, the clock propagation and charge transfer times. Lazovsky et al. ${ }^{\mathrm{xi}}$ demonstrated that $10^{8}$ frames per second are achievable in this architecture. Shuttering may either be tied to the basic clock cycle or may be achieved with an independently clocked gating layer ${ }^{\mathrm{xi}}$. After all storage registers are filled the data is readout at reduced speed, as a video or video like sequence. In this architecture the number of pixels, frames and dynamic range all trade off area on the chip. 


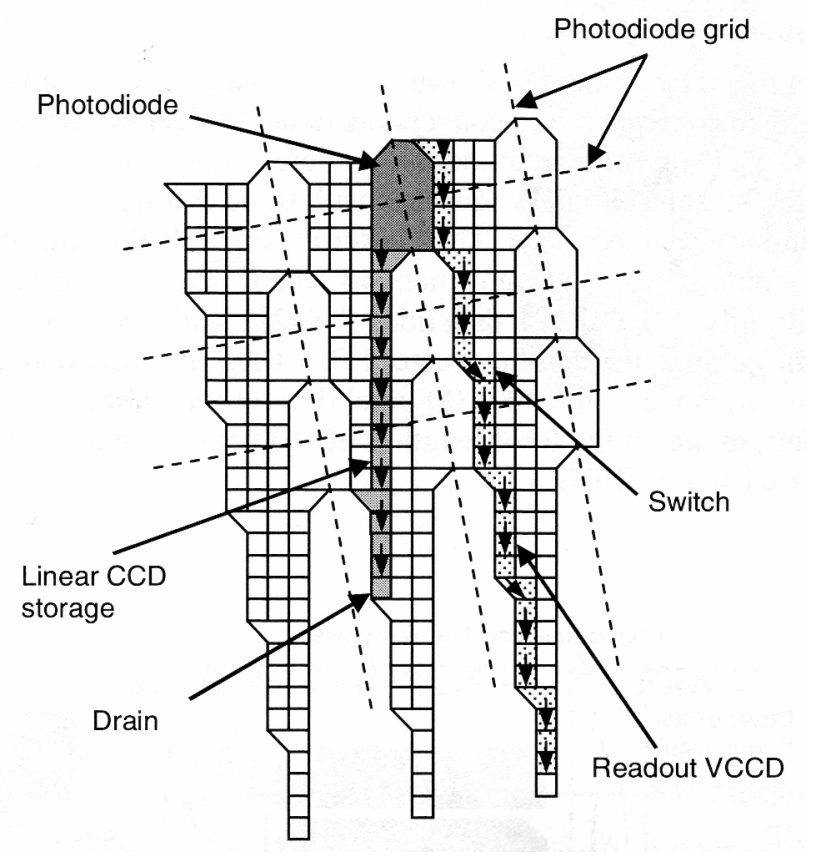

Fig. 2 Schematic of the ISIS architecture ${ }^{\text {xiii }}$. (Ref. Maruyama et al. Proc. $26^{\text {th }}$ ICHSPP, SPIE Vol 5580, 2004)

\section{Camera Evaluation}

The first commercial ISIS camera is the Shimadzu HPV-1 ${ }^{\text {xiv }}$ and LLNL was privileged to test a prototype camera. The camera was used in the field at several LLNL facilities and was bench tested both at LLNL and by the Bechtel Nevada group in Livermore ${ }^{\mathrm{xv}}$. The HPV-1 camera has a resolution of 312x260 pixels. The sensor is a front illuminated CCD with $9 \mu \mathrm{m}$ sensors elements on $66 \mu \mathrm{m}$ centers. It has a maximum frame rate of 1 mega-frame per second and a minimum exposure time of $250 \mathrm{~ns}$, storing 100 frames on the chip.

Bench testing of the camera, done at Bechtel, used a calibrated Optoliner with a $10 \mu$ s xenon flash. Various inter-frame times up to four millisecond were used to integrate the entire output of the flash within a single frame (frame 50) of the HPV-1. Data was analyzed using Princeton Instruments code WinView32. Response curves were measured monochromatically, at 550nm, and were averaged over all pixels in the array. Neutral density filters were used to vary the flash intensity (Figure 3).

Tests were also conducted at LLNL (Figure 4). A photographic strobe with a rise time of $35 \mu$ s was observed by the HPV-1 running at $10^{6}$ frames per second. The light from the strobe was reflected from a diffuse surface and was incident directly upon the sensor chip. No lens was used. The temporal light curve of the strobe was measured with a calibrated photopic response photodiode located adjacent to the imager. Thus the response was measured dynamically in a single flash lamp pulse. A third test using a uniform light box and calibrated step wedge was also used to measure dynamic range for continuous illumination. The three tests were conducted, independently, by different individuals. The Bechtel and both LLNL tests concluded that the maximum realized dynamic range was between 32 and 36 at the lowest gain settings. Higher gain settings increase contrast but reduce useable dynamic range. 
a)

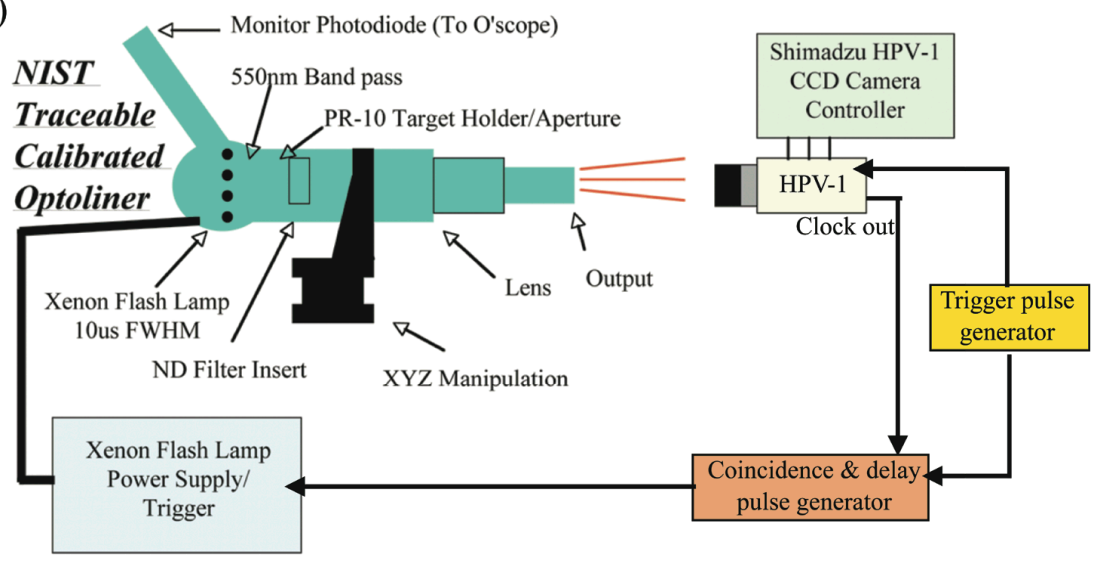

b)

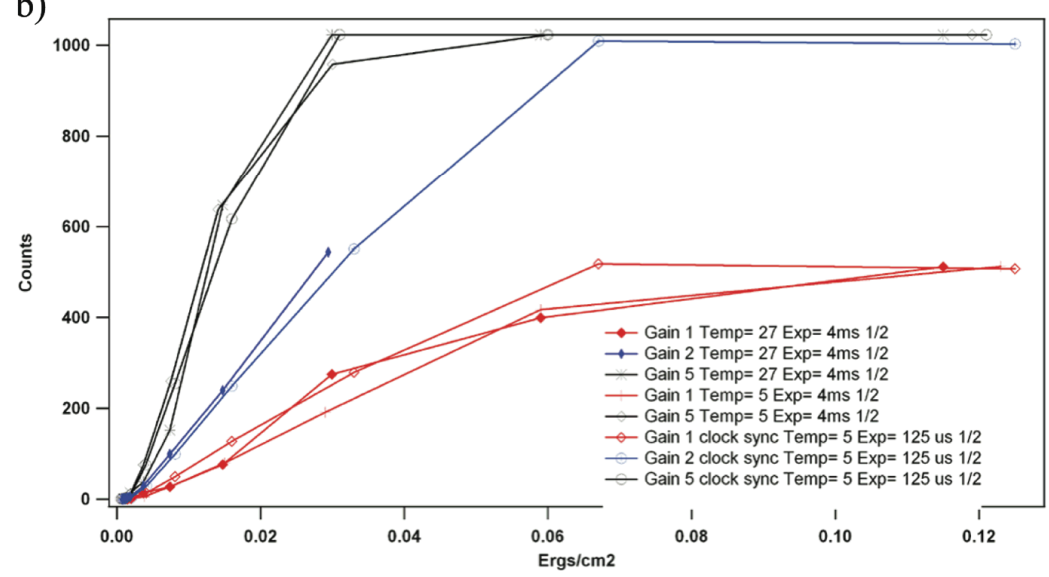

Fig. 3 a) Test configuration and b) plot of camera response vs. incident energy. The response was averaged over all pixels.

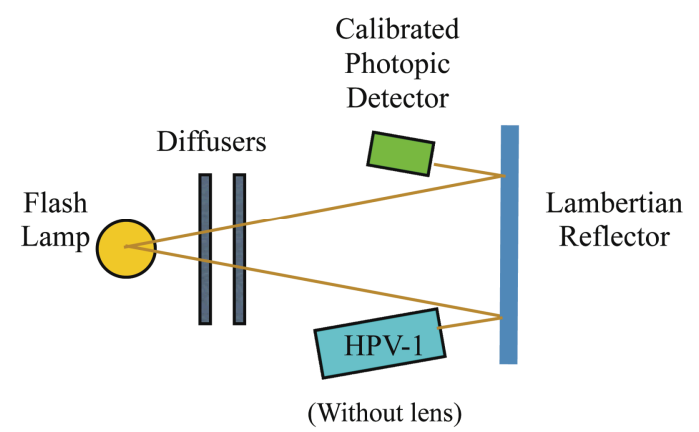

Fig 4. Experimental arrangement for Photopic Calibration

There seem to be some minor nonlinearities in the response of the camera. For gain 1 the output count is clipped at 512, whereas, at gain 2 and above it was 1024. This level clipping was also evident when data was output in the 8 bit Tiff format. There also was a difference in response from the two halves of the chip such that the response curve was considerably altered at signal levels above half saturation. These nonlinearities made it difficult to measure the full saturation level. The measurements indicated full well saturation at about $.06 \mathrm{ergs} / \mathrm{cm}^{2}$ monochromatically at $550 \mathrm{~nm}$ and photopic saturation about $1.1 \times 10^{-2} \mathrm{Lux}-$ seconds. The photopic response compared to the saturation level of Kodak Ektachrome P-1600 film $\left(3.16 \times 10^{-2}\right.$ Lux-Sec), indicates an equivalent ASA of about 4500 for the HPV-1 imager. There was also 
little difference in the noise with respect to frame rate or chip temperature, indicating the dominance of readout noise.

Resolution at the detector was measured with the Optoliner, at 7 line pairs per mm on the detector. This is consistent with the Nyquist limit of the $66 \mu \mathrm{m}$ pixel spacing for a square wave bar chart. The wide spacing of the detector elements allows the use of small lens apertures without loss of resolution.

An interesting consequence of the ISIS architecture is that blooming appears both in space and time.

Temporal blooming into adjacent frames was noted at full well saturation independent of gain setting. Spatial blooming often appeared as a pixel by pixel checker board pattern adjacent to saturated areas in the image and was evident in the field testing.

\section{Field Testing}

The field testing allowed side by side comparisons Shimadzu HPV-1, Photron APX-RS and Cordin 121 cameras (Figure 5). The Photron is currently one of the fastest continuous readout cameras but is not a mega-frame per second camera. The Photron resolution drops below the Shimadzu at frame rates faster than 20 kilo-frames per second, with the pixel count dropping proportionally with increasing frame rate. The testing also allowed the use of the HPV-1 at several facilities with many different illumination sources. The high sensitivity and the ability to use fast $35 \mathrm{~mm}$ camera lenses, allowed the use of day light at the maximum speed of the camera. However, to preserve depth of field small lens apertures (f/16-32) are preferred, thus requiring artificial lighting. Both Xenon and Argon flash lamps were used. They generally had flash durations from 30 to over 100 microseconds depending on the particular power supply. Flash bulbs were used to record slower events lasting milliseconds. The ability to change the frame rate of the Shimadzu camera also changes the exposure time. Slowing the frame rate late in the event allowed us to push into the tail of a flash source.
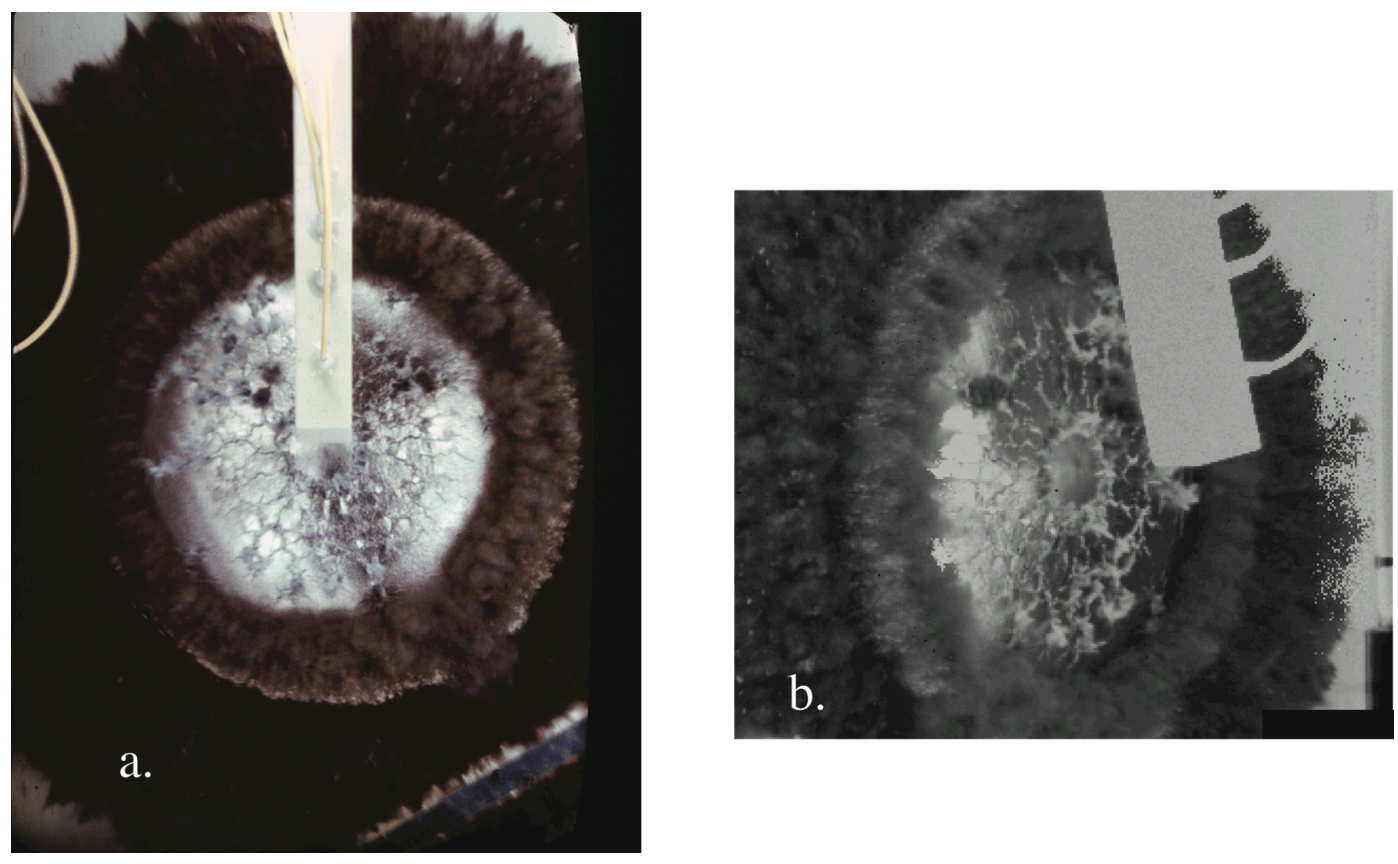

Fig. 5. Single frames from an explosively driven plate breakup experiment viewed simultaneously with;

a.) Cordin 121 and b.) Shimadzu HPV-1 cameras. The test was viewed through different lines of sight. Illumination used Mega Sun lamps ${ }^{\mathrm{xvi}}$. The white bar across the center is a support for fiber optic Velocimeter probes.

Synchronizing the camera with a short pulse laser requires using a coincidence signal with the clock output of the camera. Initially, a stochastic \pm 1 frame jitter in the triggering of the HPV-1 was found when using 
the same trigger for a laser. At times the laser pulse was lost in the dead time between frames. The coincidence technique reduced the jitter to a few nanoseconds.

A more vexing issue is that the HPV-1 cannot be started electronically. The camera is designed to be started manually and record data continuously until receiving an electronic trigger. The trigger then stops the recording and the last 100 frames are read out. The problem is that at the fastest speeds the chip heats too rapidly and the camera is designed to shut down automatically to prevent thermal over load. This precludes remote unmanned operation and makes synchronization with rotating mirror cameras unreliable.

\section{Conclusions}

The comparative advantages and disadvantages of the rotating mirror Cordin 121 and solid state Shimadzu HPV-1 cameras are given in Table 1. The issues for which the solid state mega-frame per second camera are still at a significant disadvantage, are resolution (pixel count), realized dynamic range and blooming. These issues are largely concerned with the design and area of the chip. The HPV-1 is slower than the Cordin but not significantly so. The Lazovsky ${ }^{\text {xvi }}$ paper indicates significantly higher speeds are feasible within the ISIS architecture.

\begin{tabular}{|c|c|c|}
\hline & Cordin |2| & Shamadzu HPV-I \\
\hline Resolution & $\begin{array}{c}25 \times 60 \mathrm{~mm} @ 14 \mathrm{Ip} / \mathrm{mm} \\
(\sim 2100 \times 5000 \text { pixel equivalent) }\end{array}$ & $260 \times 312$ pixels \\
\hline Frame Count & 25 & 100 \\
\hline Speed & $2.5 \mathrm{Mf} / \mathrm{s} 200 \mathrm{~ns}$ shutter & I.Mf/s, 250ns shutter \\
\hline Dynamic Range & $\begin{array}{c}100 \text { to }>10000 \\
\text { depending on film \& process }\end{array}$ & 36 \\
\hline Sensitivity & Typically ASA 1600 & ASA $\sim 4500$ \\
\hline Optical acceptance & $f / 18-36$ & $f / 1.2$ \\
\hline Blooming & Minimal & Severe \\
\hline Synchronization & Mechanical & Electronic \\
\hline Interframe alignment & No & Yes \\
\hline Data Processing & Darkroom & Electronic \\
\hline Ease of use & $\begin{array}{l}\text { Requires special } \\
\text { facilities \& training }\end{array}$ & $\begin{array}{l}\text { Use anywhere } \\
\text { Minimal training }\end{array}$ \\
\hline
\end{tabular}

Table 1 Comparative advantages (green) and disadvantages (red) of tested cameras.

The limiting visual resolution of the Cordin 121 has been measured at speed to be about 14 line pairs per millimeter across a $25 \times 60 \mathrm{~mm}$ image on the film. There is no simple conversion from line pairs to pixel count, as they are defined very differently. The definition of line pairs of resolution includes information about the dynamic range of the detector and the contrast of the object. The Nyquist Theorem requires 2.3 samples to resolve a periodic stricture. As most objects of interest are not periodic, multipliers of 6-9 are a more realistic conversion from line pairs to pixels. Using 6 pixels per line pair the Cordin resolution is comparable to a 10 Mega-Pixel array. An ISIS array of this magnitude is not feasible in the foreseeable future. However, a 1 Mega-Pixel camera may be feasible and is probably sufficient for most applications. The full resolution of the Cordin is rarely needed. In those cases when it is, it would be considerably less expensive to use two, mega-pixel, cameras working at different magnifications for the overview and detail. Using the same $66 \mu \mathrm{m}$ pixel spacing as the Shimadzu camera a $66 \mathrm{~mm}$ square chip would be a mega-pixel array. Such a chip would be compatible with commercial medium format camera optics.

Realized dynamic range is the ratio in the limiting light levels, from black to white, beyond which changes of light level are not measurable. It is important to note that although well depth is the ultimate limiting factor for dynamic range, the real limit is usually determined by the noise. The realized dynamic range is invariably less than the full well depth. Dynamic range can be improved at the chip level by increasing the well depth. This must be matched in the camera design with chip cooling and low noise analog to digital conversion to allow the translation of deep wells into realized dynamic range. Thermal noise depends on 
the temperature of the chip and the time it takes from when the pixel is recorded to when it is read out. Readout noise is created in the analog to digital converter and is a function of the speed at which the chip is read out. For most one shot experiments the readout time is irrelevant. Thus, the camera design philosophy should be to use a high dynamic range $\mathrm{A} / \mathrm{D}$ as slowly as necessary to minimize the readout noise. Then cool the chip appropriately to minimize the thermal noise buildup.

In solid state cameras, once pixels saturate, they can dump excess charge, bloom, into adjacent sites forcing them into saturation as well. In the ISIS architecture adjacent sites are storage sites of nearby pixels stored previously. Thus, unique to ISIS, blooming occurs in both image space and time. The bench tests performed on the HPV-1 were sensitive only the temporal blooming as the entire chip was illuminated uniformly. The tests indicated the onset temporal of blooming at or near saturation. Spatial blooming was quite evident in the field tests (Figure 6). In film there is an antihalation layer on the back surface to absorb excess light thus blooming effects are minimal.

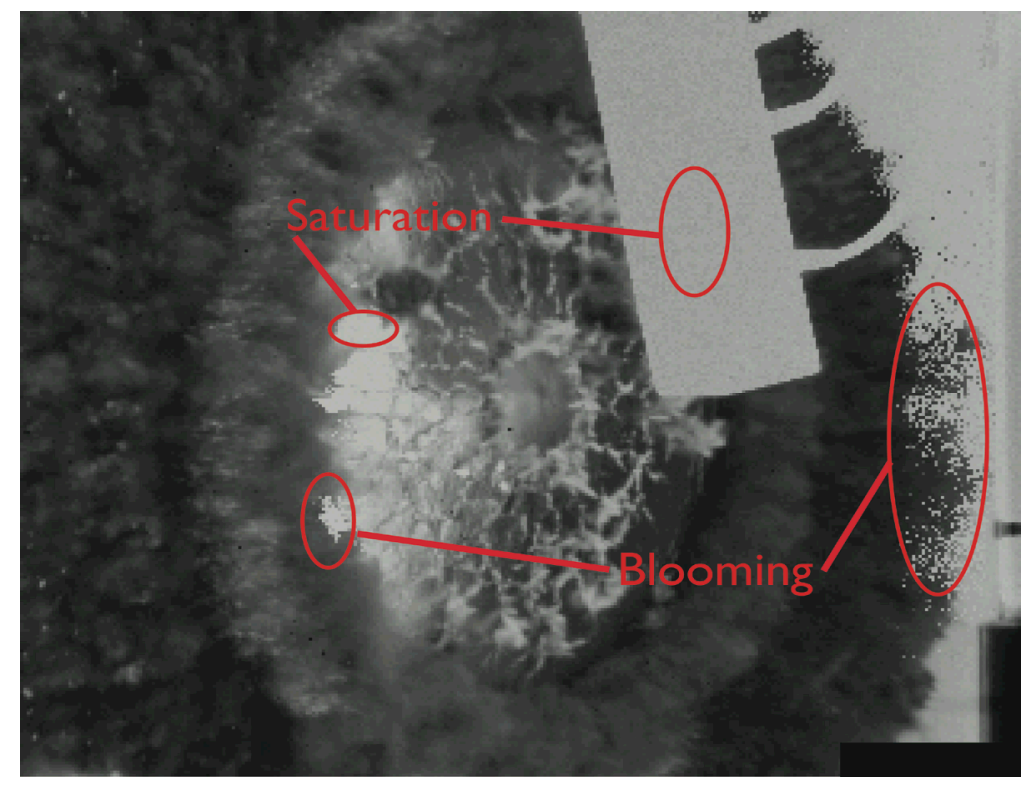

Fig. 6. Single HPV-1 frame of plate breakup experiment, showing effects of saturation and blooming. Note different white levels in saturated areas on left and right side of image.

The Shimadzu camera provides excellent images when the illumination of the scene precisely matches the dynamic range of the camera (Figure 7). However, in materials dynamics testing it is often impossible to control or predict the light from the image. Test exposures before experiments often don't give sufficient information to determine the appropriate exposure settings and with expensive one-shot experiments there is no second chance. Most experimental surprises occur from unexpected light either from dynamic reflections or self light from the experiment. The solution is to have sufficient dynamic headroom and protection from blooming.

The Shimadzu's 100 frame capability is excessive for many applications, especially with the capability of changing recording speeds in real time. Thus reducing the frame count would free area on the chip for both deeper wells and anti-blooming electrodes without having to increase pixel spacing.

It is interesting to note that current development in Japan ${ }^{\text {xviii }}$ appears to be concentrating on commercial TV sports. For this application the chip and camera design trade-offs are quite different. Lighting is generally constant thus much less dynamic range is needed. Fast readout (30 frames per second) and maximum frame count are essential. HDTV resolution and format are desired as well as a chip size compatible with $35 \mathrm{~mm}$ camera optics. 
At the present time there is no fully solid state replacement for the mega frame per second rotating mirror framing cameras. It is clear, however, that the ISIS architecture has the potential to provide this capability. To develop an appropriate camera both the chip and camera designs will have to be optimized specifically for this function.

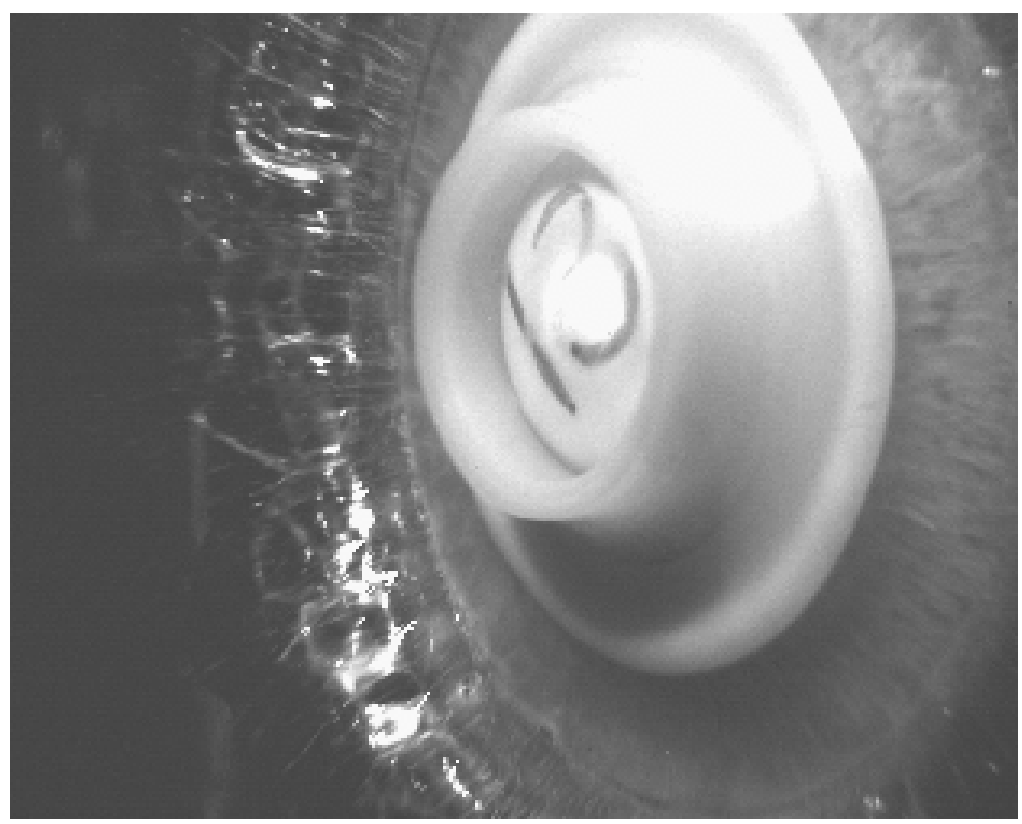

Fig.7. Single HPV-1 frame from gas gun projectile impact illuminated by single FT-34 flash lamp. Some minor blooming is evident in splash area.

\section{Acknowledgements}

The authors would like to acknowledge M. Takaki and Y. Kondo of Shimadzu Corporation for providing the HPV-1 camera for evaluation, and C. Diamond, T. Bilir, V. Tran \& J Cradick, of Bechtel Nevada, Livermore Operations for their support in bench testing the camera. 


\section{References}

${ }^{\mathrm{i}}$ This work was performed under the auspices of the U.S. Department of Energy by the University of California, Lawrence Livermore National Laboratory under contract No. W-7405-Eng-48.

ii $T$. G Etoh et. al. Proc. $24^{\text {th }}$ ICHSPP, SPIE Vol 4183, 2000

iii Shimadzu Corp. 'Hypervision', Model HPV-1

${ }^{\text {iv }}$ Haddleton, G.P., Proc. $25^{\text {th }}$ ICHSPP, SPIE Vol 4948, 2002

${ }^{v}$ Boys, C. V., Nature, 47, 415, 440, London, 1893

${ }^{v i}$ Miller, C. D., J. Soc. Mot. Pict. Engrs. 53, 479, 1949

${ }^{v i i}$ Brixner, B, J. Opt. Soc. Amer., 45, 876, 1955

viii The mention of specific manufacturers or products is for reference only and does not represent an endorsement by the authors or Lawrence Livermore National Laboratory.

${ }^{\text {ix }}$ LLNL does not specifically endorse any commercial product.

${ }^{\mathrm{x}}$ W.F. Kosnocky \& J.L. Lowrance, U.S. Patent 5355 165, Oct. 11, 1994.

${ }^{x i}$ L.Lazovsky, D. Cismas, G. Allan \& D. Given, Proc. SPIE, Vol. 5787, 2005

${ }^{x i i}$ R.K. Reich, R.W. Mountain, W.H. McGonagle, J.C-M. Huang, J.C. Twichell, B.B. Kosicki, and E.D. Savoye, "Integrated Electronic Shutter for Back-illuminated Charge-Coupled Devices," IEEE Transactions on Electron Devices, Vol. 40, no. 7, July 1993, pp.1231-1237.

${ }^{x i i i}$ Maruyama et al. Proc. $26^{\text {th }}$ ICHSPP, SPIE Vol 5580, 2004

${ }^{\text {xiv }}$ LLNL, op. cit.

${ }^{x v}$ Private communication, C. Diamond, T. Bilir \& J Cradick, Bechtel Nevada Livermore Operations.

${ }^{x v i}$ R.G.Root, Proc. $25^{\text {th }}$ ICHSPP, SPIE Vol 4948, 2002

${ }^{x v i i}$ L.Lazovsky et. al. op. cit.

xviii T. G Etoh, private communication. 